University of Nebraska - Lincoln

DigitalCommons@University of Nebraska - Lincoln

Virology Papers

Virology, Nebraska Center for

8-2019

\title{
Longitudinal quantification of adenovirus neutralizing responses in Zambian mother-infant pairs: Impact of HIV-1 infection and its treatment
}

Sara R. Privatt

Brianna L. Bullard

Eric A. Weaver

Charles Wood

John T. West

Follow this and additional works at: https://digitalcommons.unl.edu/virologypub

Part of the Biological Phenomena, Cell Phenomena, and Immunity Commons, Cell and Developmental Biology Commons, Genetics and Genomics Commons, Infectious Disease Commons, Medical Immunology Commons, Medical Pathology Commons, and the Virology Commons

This Article is brought to you for free and open access by the Virology, Nebraska Center for at DigitalCommons@University of Nebraska - Lincoln. It has been accepted for inclusion in Virology Papers by an authorized administrator of DigitalCommons@University of Nebraska - Lincoln. 


\title{
Longitudinal quantification of adenovirus neutralizing responses in Zambian mother-infant pairs: Impact of HIV-1 infection and its treatment
}

\author{
Sara R. Privatt, Brianna L. Bullard, Eric A. Weaver, \\ Charles Wood, and John T. West
}

Nebraska Center for Virology and School of Biological Sciences,

University of Nebraska, Lincoln, Nebraska, USA

Correspondence - J.T. West, jwest2@unl.edu

\begin{abstract}
Vaccination offers the most cost-effective approach to limiting the adverse impact of infectious and neoplastic diseases that reduce the quality of life in sub-Saharan Africa (SSA). However, it is unclear what vaccine vectors would be most readily implementable in the setting and at what age they should be applied for maximal efficacy. Adenoviruses (Ad) and Ad-based vectors have been demonstrated to induce effective humoral and cellular immune responses in animal models and in humans. However, because immunity associated with Ad infection is lifelong, there exists a debate as to whether pre-existing immunity might decrease the efficacy of Ad vectored vaccines. In order to begin to rationally develop vaccination strategies for SSA, we have quantified neutralizing antibodies (nAb) against Ad4, Ad5, Ad7, Ad26, Ad28, Ad45 and Ad48 in 67 adult women and their infants. We are the first to define the decay kinetics of transferred maternal nAb in infants as well as the

Published in Vaccine 37 (2019) 5177-5184

doi:10.1016/j.vaccine.2019.07.074

Copyright (C) 2019 Elsevier Ltd. Used by permission.

Submitted 30 May 2019; revised 17 July 2019; accepted 23 July 2019;published 1 August 2019.
\end{abstract}


apparent initiation of de novo Ad responses. Our findings demonstrate that in Zambian adults, robust nAb responses exist against each of the Ads tested and are efficiently transferred to newborns. With few exceptions, neither the HIV-1 infection status of the mothers or the antiretroviral therapy (ART) treatment of HIV-1 disease had significant impact on maternal Ad nAb responses or their transfer to infants. However, maternal Ad nAb decays in infants to a nadir at 12 months of age such that any of the seven Ad types could function as vaccine vectors. The definition of this 'window of opportunity' provides important foundational data for rational design and implementation of Ad vectors in this setting.

Keywords: Adenovirus, Seroprevalence, Vector, Sub-Saharan Africa, HIV

\section{Introduction}

Sub-Saharan Africa (SSA) is disproportionately affected by infectious diseases. The World Health Organization (WHO) has reported that two thirds of people living with HIV are in SSA [1]. Similarly, there is increased prevalence of tuberculosis, malaria, and other communicable diseases, as well as higher incidence of infection-related cancers. The incidence of infectious disease associates with significantly higher mortality in comparison to developed regions [2-4]. The public health impact of infectious diseases in SSA highlights the need for a more rational appreciation of vaccine designs with potential to reduce the regional disease burden. One important issue with vaccine design is the selection of suitable vector for the delivery of the vaccine, especially for infants in SSA, where effective childhood vaccinations could decrease disease incidence.

One promising investigational vaccine platform is Adenovirus (Ad). Adenoviruses are large double stranded DNA viruses that, through deletion of genetic content and propagation to high titers, can be used as gene-delivery tools or vaccine vectors [5]. Historically, Ad vaccines and vectors were replication competent and thus had some risk of causing an Adenoviral infection [6,7]. Modern Ad constructs are typically replication incompetent or produce single cycle infections where no new Ad particles are produced but an inserted transgene of interest is efficiently expressed [8]. These recombinant Ads have been used developmentally as vectors in many Phase 1 and 2 vaccine safety trials against a diverse cadre of pathogens, including Zika virus, HIV, influenza, and Ebola; however, safety and efficacy have only 
been demonstrated for nonrecombinant vaccines used by the US military against Ad types 4 and 7 [9-16]. Adenoviral vaccination typically produces a strong adaptive, Ad serotype-specific immune response $[12,17]$. The prevalence of pre-vaccination adenovirus-specific immunity needs to be considered when predicting potential efficacy for vaccination in infants and young children. For example, Adenovirus type 5 (Ad5) is commonly used in pre-clinical research and has been tested in human vaccine trials. Unfortunately, Ad5 has high worldwide seroprevalence suggesting that gene-delivery strategies based on that subtype could suffer from decreased effectiveness due to pre-existing neutralizing antibody (nAb) responses to the vector $[18,19]$. Consistent with this concept, the HIV-1 STEP trial, which utilized an Ad5 backbone to deliver the genes for HIV-1 gag, pol, and nef proteins as potential immunogens, was prematurely discontinued when a correlation was observed between pre-existing Ad5 immunity and new HIV-1 infections [20]. However, data from non- HIV targeted Ad vaccine efforts, as well as studies using other viral vector systems, such as measles or dengue, have demonstrated preclinical and clinical efficacy in the face of pre-existing neutralizing responses [21-24]. Thus, the impact of pre-existing Ad nAb on increased infection may be a unique function of HIV biology since it has not been consistently reported by other groups utilizing various vaccine targets delivered by Ad vectors [24].

There are currently more than 60 serotypes of circulating Ads, separated into 7 subspecies, A-G, with varying geographical prevalence [25]. Adenoviruses are categorized based on infection associated conditions, serology, propensity to cause tumors in rodents, and genome sequence. Here, we focus on the humoral immune responses to Ads from subgroups B-E. Adenovirus type D is the largest of the subspecies and most newly discovered Ads fit into this category, yet most research has been performed on types B, C, and E [26,27]. Subspecies B, C, and E adenoviruses are typically associated with acute respiratory infections and conjunctivitis which can cause serious disease in immunocompromised individuals [28,29]. However, very little is known about the adenoviral serotypes that are commonly found in Africa, and a better understanding of the existing immune response against adenovirus is important for the designing of vaccine which can be used in the setting. We have included four different type D viruses, 
Ad26, Ad28, Ad45 and Ad48, to expand on the limited knowledge of this subgroup. These adenovirus subspecies typically associate with respiratory diseases and conjunctivitis in humans and are likely responsible for many of the respiratory infections found in Africa and elsewhere $[28,30]$.

In the current study, we characterized the humoral immune response against seven different adenovirus serotypes in two Zambian cohorts consisting of mother-infant pairs collected at different time points. Many of the mothers were also HIV-1 infected. Investigation of immune responses in these cohorts will not only provide important information on the circulating adenoviral serotypes and the humoral immune response against adenovirus in the Zambian population, but also provide a better understanding of the pre-existing mother to infant transmission of antiadenovirus antibodies, and the impact of untreated and treated HIV-1 infection on immune responses to adenoviral infections. Moreover, with the longitudinal infant follow-up specimens, we investigated the decay kinetics of maternal neutralizing antibodies ( $\mathrm{nAb}$ ) in infants and characterized the nadir of Ad seroreactivity prior to de novo response in those same infants. This information could form the foundation for rational selection of adenoviral gene delivery platforms for prophylactic or therapeutic application.

Our results suggest that in Zambian adults, pre-existing humoral immunity exists against all 7 Ads tested and is efficiently transferred to newborns. However, due to maternal Ab decay in infants, any of the 7 Ad types could function as vaccine vectors between 12 and 24 months of age, thereby defining the 'window of opportunity' for use of Ad vectors for vaccination of children in this setting.

\section{Materials and methods}

\subsection{Cohort description and sampling}

There were two cohorts that were analyzed. The first cohort samples were collected between 1998 and 2003 in the pre-ART era as part of a prospective study investigating the effects of HIV-1 and KSHV infection among Zambian mothers and their infants at the University Teaching Hospital and the University of Zambia School of Medicine, 
Lusaka, Zambia. Written informed consent was obtained from all participants. Blood samples were collected by venipuncture and blood was collected in acid citrate dextrose tubes and processed within $6 \mathrm{~h}$ of being drawn. Samples were frozen at $-80^{\circ} \mathrm{C}$ and shipped to the University of Nebraska- Lincoln for long-term storage. Protocols were reviewed and approved by the Institutional Review Boards at the University Teaching Hospital and the University of Nebraska-Lincoln.

The second cohort samples were collected between 2010 and 2013 when ART had become available in Zambia, and they were collected as part of an observational study investigating the effect of ART on KSHV transmission. Women with infants under the age of 12 months were enrolled into this study to examine the determinants of KSHV seroconversion. This study was approved by the Institutional Review Board of the University of Nebraska-Lincoln and the University of Zambia Biomedical Research Ethics Committee. From these cohorts, a subset of 63 mother-infant pairs (MIPs) were selected based on either mother HIV-1 infection status or ART treatment status. MIPs were also selected based on infant follow-up sample availability for nAb testing.

\subsection{Recombinant adenovirus construction}

All Ad constructs contain a green fluorescent protein-luciferase (GFP-Luc) fusion gene under a CMV promoter within either the $E_{3}$ gene or between $E 1 A$ and $E 1 B$. The recombinant Adenovirus types 4 and 7 contain a GFPLUC reporter expression cassette in the $E_{3}$ gene and were constructed as described in Weaver, 2014 [31]. Recombinant Adenovirus types 26, 28, and 48 construction was described in Weaver and Barry [9]. Recombinant adenovirus type 45 was produced as previously described [31]. The recombinant $\mathrm{Ad}_{5}-855$ virus was constructed as described in [PMID: 10846098].

\subsection{Recombinant adenovirus purification}

The recombinant adenovirus genomes containing the GFP-Luc insert were linearized and buffer exchanged using a Strataprep PCR purification kit (Agilent Technologies). The linearized recombinant gDNA was transfected into HEK293T cells using the PolyFect Transfection Reagent (Qiagen). Virus infection was indicated via plaque 
formation, at which time cells were harvested and infectious progeny virus was released by 3 freeze-thaw cycles. The recombinant Ad was amplified by sequential passages in HEK293T cells prior to final amplification in a Corning 10-cell stack $\left(\sim 6300 \mathrm{~cm}^{2}\right)$. Amplified virus was purified by two sequential $\mathrm{CsCl}$ ultracentrifuge gradients and desalted using Econo-Pac 10DG desalting columns (Bio-Rad). The virus particle quantity was determined by absorption at $260 \mathrm{~nm}$ on a NanoDrop Lite Spectrophotometer (Thermo Fisher). Virus aliquots were stored at $-80{ }^{\circ} \mathrm{C}$ in Ad-tris buffer (20 mM Tris- $\mathrm{HCl}, 100 \mathrm{mM} \mathrm{NaCl}{ }_{2}$, $1 \mathrm{mM} \mathrm{MgCl} 6 \mathrm{H}_{2} \mathrm{O}, 10 \%$ glycerol). HEK293T cells were grown in Dulbecco's modified Eagle's medium supplemented with 10\% heat inactivated $\mathrm{FBS}$ and $1 \%$ penicillin-streptomycin at $37{ }^{\circ} \mathrm{C}$ and $5 \%$ supplemented $\mathrm{CO}_{2}$.

\subsection{Adenovirus neutralization assay}

Adenovirus-specific nAb activity was quantified by a reduction in luciferase activity. Briefly, maternal or infant sera were heat inactivated at $56^{\circ} \mathrm{C}$ for $60 \mathrm{~min}$. Next, $20 \mu \mathrm{L}$ of serum was added to $380 \mathrm{~mL}$ of DMEM containing $1.2 \times 10^{9}$ viral particles for a final volume of 400 $\mathrm{mL}$, resulting in a final serum dilution of 1:20. The serum/virus mixture was incubated at $37^{\circ} \mathrm{C}$ for $60 \mathrm{~min} .100 \mu \mathrm{L}$ was then added to 50 $\mathrm{mL}$ of DMEM containing $1 \times 10^{5} 293 \mathrm{~T}$ cells per well in triplicate in a 96 well plate and allowed to incubate for $24 \mathrm{~h}$. The luciferase activity was determined using the reporter lysis $5 \times$ buffer and luciferase assay reagent (LAR) system (Promega, Madison, WI). $40 \mathrm{~mL}$ of reporter lysis $5 \times$ buffer was added and allowed to incubate at room temperature for $20 \mathrm{~min}$. Neutralization was defined as percent of virus only luciferase readout, which was then subtracted from $100 \%$. We have defined neutralization as $>90 \%$, mild neutralization as $70-90 \%$ and nonneutralizing as $<70 \%$.

\subsection{Statistical analysis}

Statistical analyses were conducted using GraphPad Prism 7 software. Non-parametric Mann-Whitney tests were used to determine statistical significance. $P$ values $\leq 0.05$ were considered statistically significant. 


\section{Results}

\subsection{Maternal neutralizing antibodies against subgroups $B, C, E$ and $D$ adenoviruses}

Adenovirus seroprevalence has not been evaluated in Zambian maternal-infant pairs (MIPs); therefore, the impact of HIV-1 infection, and its treatment with ART, on (a) maternal nAb production, (b) transfer of nAb to newborns, (c) the decay of maternal Ab in neonates, and (d) the development of infant de novo nAb responses, is incompletely understood. In order to estimate the seroprevalence of adenoviruses in Zambia, we tested 42 women in a pre- ART cohort and 25 women in a post-ART cohort for $\mathrm{nAb}$ against recombinant Ad7 (Group B), Ad5 (Group C) and Ad4 (Group E) containing a GFPLuc reporter. Comparison of responses in the two temporally distinct cohorts allowed for evaluation of the change in breadth and magnitude of Ad nAb coincident with ART implementation in the region.

Other groups have defined neutralization with thresholds from $50 \%$ up to $90 \%$ [25,32-35]. We conservatively defined neutralization as $\geq 90 \%$ mean neutralization for $\mathrm{n}=3$ replicates. The number of women who neutralized Ad5 was not significantly different between the pre- and post-ART maternal cohorts, 93\% and 88\% respectively. The majority of women in the pre-ART cohort (52\%) had neutralizing activity exceeding 90\% against $\mathrm{Ad}_{4}$ and Ad7. However, only 28\% of women in the post-ART cohort exhibited nAb responses against those types. (Fig. 1).

Since species D adenoviruses were reported to be frequently isolated from immunosuppressed subjects and those with keratoconjunctivitis [36], and because this group was shown to have lower seroprevalence than Ads B, C, and E in other geographical regions, we tested for seroreactivity against four Group D Ads: Ad26, Ad28, Ad45 and Ad48 [26,27]. Given that these four Ads are members of the same Ad group, we anticipated levels of $\mathrm{nAb}$ to be similar in adults. Consistent with this concept, the majority of women in both the pre- and postART cohort did neutralize Ad26, Ad28 and Ad45 (Fig. 1). The exception was Ad48 where $<25 \%$ had detectable nAb ( $\geq 90 \%$ ), irrespective of the cohort. 

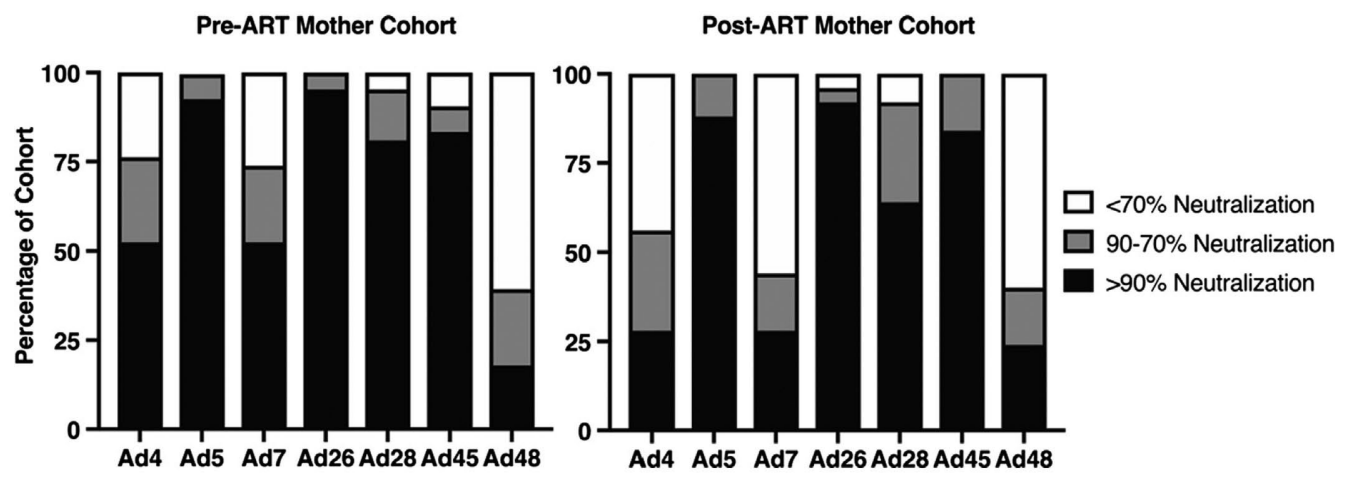

Fig. 1. Neutralization profile of adult women in Zambia, Africa against Ad4, Ad5, Ad7, Ad26, Ad28 Ad45 and Ad48. 42 serum samples from the pre-ART cohort and 25 samples from the post-ART cohort were evaluated for neutralizing activity against Ad4, Ad5, Ad7, Ad26, Ad28, Ad45, and Ad48 at a 1:30 sera dilution by luciferasebased neutralization assay. Luciferase inhibition indicates viral neutralization by sera. Each sample was placed into one of three categories based on neutralization percentage compared to virus only samples: $>90 \%, 90-70 \%$ or $<70 \%$ which equates to highly neutralizing, mildly neutralizing, or non-neutralizing, respectively.

\subsection{Transfer of maternal $n A b$ and the impact of HIV infection in the pre-ART cohort}

To evaluate patterns of maternal pre- and perinatally nAb transfer to infants, the percent neutralization for each adenovirus was quantified in sera collected from infants in 6-month intervals starting on the day of birth. The MIPs in the pre-ART cohort were recruited between 1998 and 2003, a time when Zambian women did not readily have access to ART treatment. Comparisons of the median nAb responses against each Ad subtype are shown in Fig. 2A and B. Median infant $\mathrm{nAb}$ responses were significantly lower than maternal against Ad4, Ad7, Ad26 and Ad28. In contrast, similar maternal and infant neutralizing responses were detected against $\mathrm{Ad}_{5}$ and $\mathrm{Ad} 45$. The median neutralization activity in infants against Ad5 and $\operatorname{Ad} 45$ was $99 \%$ and $99 \%$, respectively. Because 4 of 28 maternal sera enhanced infection, median neutralizing activity against $\mathrm{Ad} 48$ was not significantly different between mothers and infants. These findings suggest that pre-existing immunity could lower the effectiveness of a vaccine vectored with Ad5 or Ad45 if administered on the day of birth. 


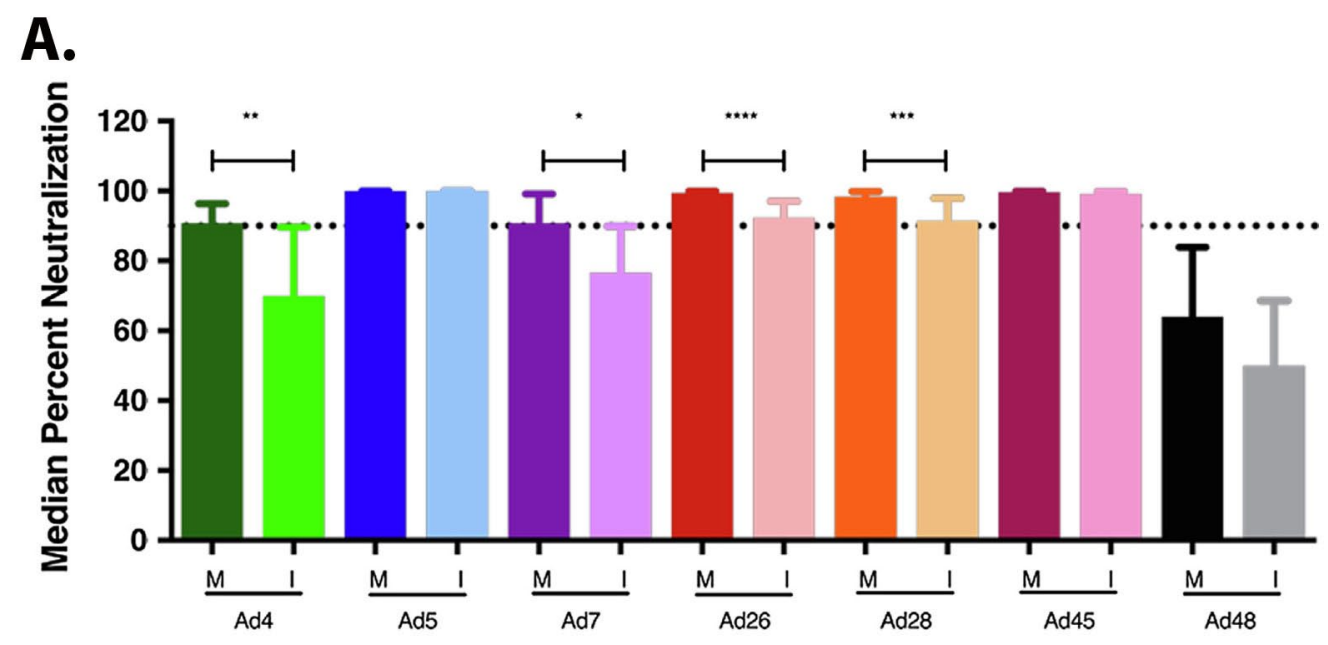

B.

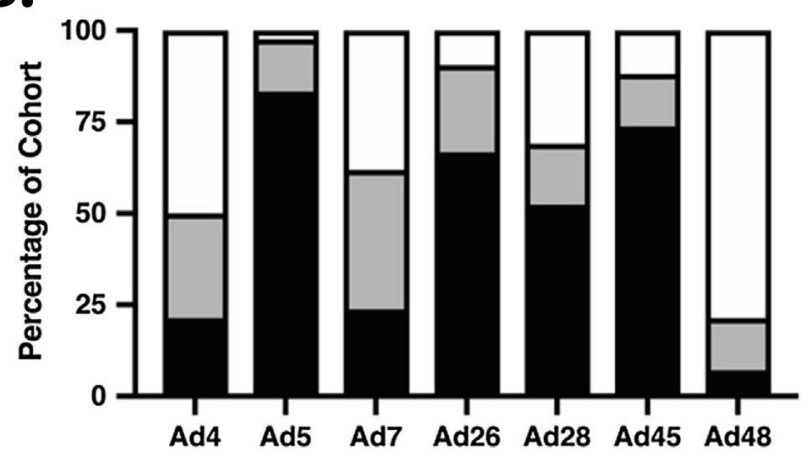

$\square<70 \%$ Neutralization
$\square>0-70 \%$ Neutralization
$>90 \%$ Neutralization

Fig. 2. Mother to Infant transfer of neutralizing antibodies in pre-ART cohort. A. Mother to infant $\mathbf{n A b}$ transfer in the pre-ART cohort. Neutralizing antibody transfer was evaluated by a comparison of total medians of percent neutralization between mother and infant pairs on day of birth. Statistical significance was determined by nonparametric Mann-Whitney tests. B. Infant neutralization profile in the pre-ART cohort. Each infant sample was placed into one of three categories based on neutralization percentage compared to virus only samples: >90\%, 90$70 \%$ or $<70 \%$ which equates to highly neutralizing, mildly neutralizing, or nonneutralizing, respectively $\left(* \mathrm{P} \leq 0.05,{ }^{*} \mathrm{P} \leq 0.01,{ }^{*} * \mathrm{P} \leq 0.001,{ }^{*} * * \mathrm{P} \leq 0.0001\right)$.

The pre-ART cohort was divided into two groups based on the HIV-1 status of the mothers, while the infants, irrespective of their infection status, were correspondingly grouped with their mothers. No significant difference was seen in overall maternal nAb activity between HIV-1 infected and uninfected women (S1). Table 1 shows that maternal HIV-1 infection significantly reduced transplacental transfer of Ad nAb only for subtypes Ad7 and Ad26. 
Table 1 Impact of maternal HIV infection on passive transfer of nAb on day of birth. For each MIP, the percent neutralization of the infant was divided by that of the cognate mother. The average of those ratios is reported for HIV-1 negative mothers (Column 2) versus positive (Column 3). Differences in the average of ratios based on HIV-1 status was statistically evaluated using non-parametric Mann-Whitney tests. A significant difference based on HIV-1 infection status was evident against Ad7 and Ad26 ( ${ }^{*} \mathrm{P} \leq$ 0.05).

\begin{tabular}{lcccc} 
Adenovirus & HIV- & $H I V+$ & Significance & $P$-Value \\
\hline Ad4 & 0.86 & 0.96 & $n s$ & 0.230 \\
Ad5 & 1.00 & 1.00 & $n s$ & 0.914 \\
Ad7 & 0.95 & 0.87 & $*$ & 0.048 \\
Ad26 & 0.96 & 0.91 & $*$ & 0.039 \\
Ad28 & 0.94 & 0.94 & $n s$ & 0.907 \\
Ad45 & 1.00 & 0.99 & $n s$ & 0.995 \\
Ad48 & 0.63 & 0.71 & $n s$ & 0.658 \\
\hline
\end{tabular}

\subsection{Transfer of maternal neutralizing antibodies and impact of ART treatment in post-ART cohort}

In the post-ART cohort, infants received significantly less maternal $\mathrm{nAb}$ against all 7 tested adenoviruses compared to the median values from the pre-ART cohort (Fig. 3A and B). The highest median neutralization in infants was against $\mathrm{Ad}_{5}$ at 93\%, a value significantly lower than that from the pre-ART cohort (99\%), but above the neutralization threshold. Median infant neutralizing activity against all other adenoviruses was below 90\%, and is therefore non-neutralizing or lacking in pre-existing immunity.

To investigate whether or not ART treatment impacted the extent of nAb transfer to infants, the post-ART cohort was separated into ART-treated and -untreated mothers along with their corresponding infants. HIV-1 treated and untreated women showed no significance in overall maternal nAb activity (S1). Differential perinatal transfer was only evident for Ad5 nAb. This finding suggests that the overall decrease in Ad nAb in post-ART infants was not associated with ARTmediated maternal immune reconstitution (Table 2). 


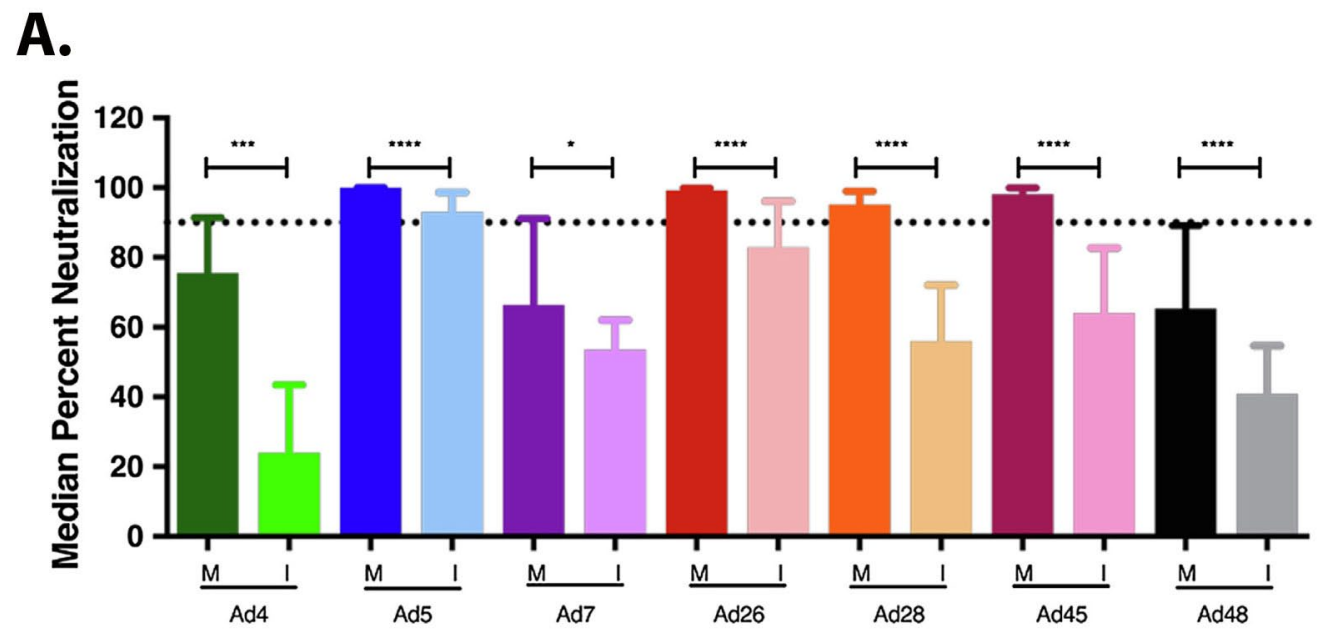

B.

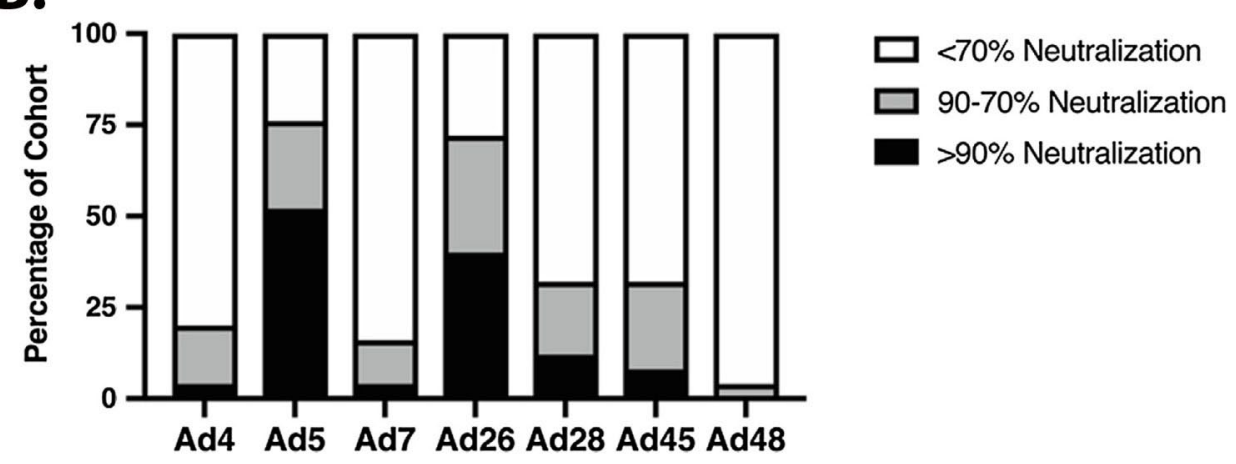

Fig. 3. Mother to Infant transfer of neutralizing antibodies in post-ART cohort. A. Mother to infant nAb transfer in post-ART cohort. Neutralizing antibody transfer was evaluated by a comparison of total medians of neutralization between mother and infant pairs on day of enrollment (ENR) in the study. Infant ENR timepoint includes infants between $\mathrm{O}$ and 3 months of age. Statistical significance was determined by non-parametric Mann-Whitney tests. B. nAb profile of post-ART cohort infants. Each infant sample was placed into one of three categories based on neutralization percentage compared to virus only samples: $>90 \%, 90-70 \%$ or $<70 \%$ which equates to highly neutralizing, mildly neutralizing, or non-neutralizing, respectively $\left({ }^{*} \mathrm{P} \leq 0.05,{ }^{* * *} \mathrm{P} \leq 0.001,{ }^{* * * *} \mathrm{P} \leq 0.0001\right)$. 
Table 2 Effect of maternal ART treatment on transfer of nAb from mother to infant. For each MIP, the percent neutralization of the infant was divided by that of the cognate mother. The average of those ratios is reported for ART positive mothers (Column 2) versus negative (Column 3). Differences in the average of ratios based on treatment status was statistically evaluated using non-parametric Mann-Whitney tests. A significant difference based on ART treatment status was evident only against $\operatorname{Ad}_{5}(* \mathrm{P} \leq 0.05)$.

\begin{tabular}{lcccc} 
Adenovirus & ART+ & ART- & Significance & $P$-Value \\
\hline Ad4 & 0.47 & 0.45 & ns & 0.799 \\
Ad5 & 0.97 & 0.80 & $*$ & 0.021 \\
Ad7 & 0.79 & 0.72 & ns & 0.903 \\
Ad26 & 0.88 & 0.89 & ns & 0.249 \\
Ad28 & 0.69 & 0.59 & ns & 0.217 \\
Ad45 & 0.65 & 0.63 & ns & 0.562 \\
Ad48 & 0.69 & 0.60 & ns & 0.411 \\
\hline
\end{tabular}

\subsection{Transfer of $n A b$ between mothers and infants coincident with ART rollout}

To quantify the differentials in maternal nAb transfer to infants between the pre- and post-ART cohorts, we calculated the median ratios of infant to mother neutralization after conversion of those ratios as a percentage. We found that transferred nAb against all Ads, excepting Ad48, was significantly reduced in post-ART cohort infants (Fig. 4). Ad 48 also showed lower nAb, but the difference was not significant. Since only modest variation in maternal Ad4 and Ad7 neutralization was detected in response to HIV-1 infection or its treatment, this data suggests that neither variable altered the extent of nAb transferred to infants.

\subsection{Degradation of neutralizing antibodies over time}

In order to determine whether an optimal age for Ad-vectored infant vaccination might exist, and which Ad subtype(s) platform would be most efficacious, we quantified infant Ad nAb over time. Starting form from initial levels near those of the maternal donor, infant $\mathrm{nAb}$ levels decreased starting at 6 months after birth (Fig. 5A and B). All 


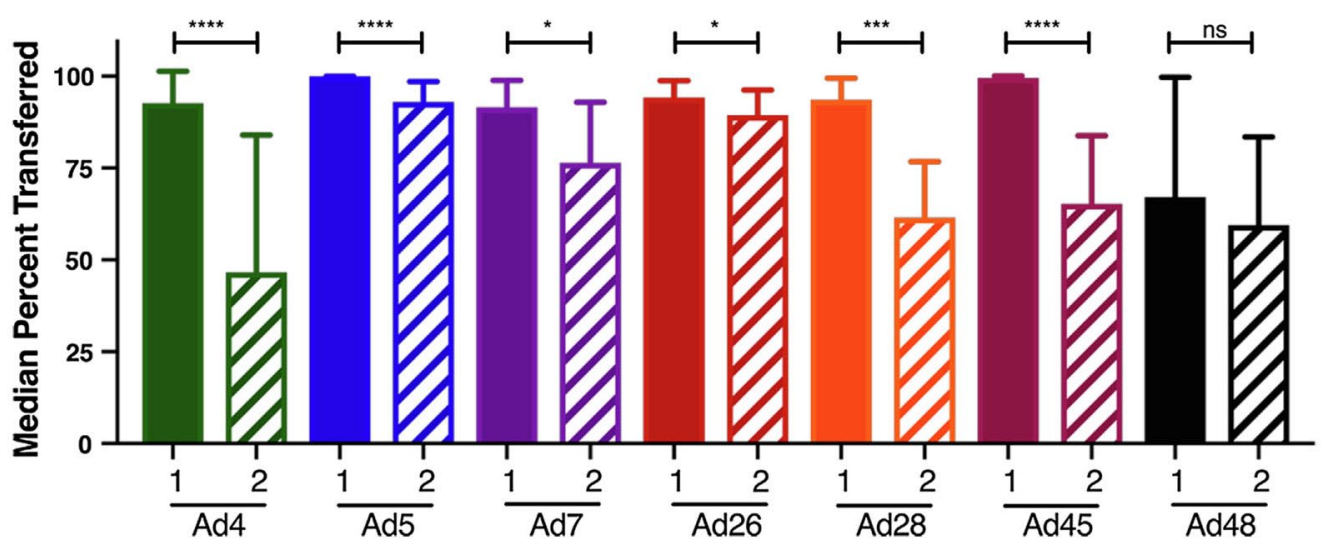

Fig. 4. Maternal to Infant nAb Transfer in pre-ART (1) and post-ART (2) cohorts. An analysis of the change in nAbs transferred to infants over time was done by comparing the fold change between the pre-ART and post-ART cohorts. Fold change was calculated by dividing the infant neutralization percentage by that of the corresponding mother neutralization percentage. The medians are shown and MannWhitney tests were used to analyze statistical significance $(* \mathrm{P} \leq 0.05, * * * \mathrm{P} \leq 0.001$, $* * * * \mathrm{P} \leq 0.0001)$.

groups demonstrated a median neutralization below $70 \%$ at 12 months regardless of cohort or Ad. In both the pre and post-ART cohorts, the highest median neutralization was against Ad5 at 68.6\% and 52.5\% respectively. The lowest median $\mathrm{nAb}$ response was seen against $\mathrm{Ad} 48$ (26.1\%) in the pre-ART cohort and against Ad4 (8.5\%) in the postART cohort.

In the pre-ART cohort, we were able to follow the $\mathrm{nAb}$ response against $\mathrm{Ad}_{4}, \mathrm{Ad}_{5}$, and Ad7 out to 36 months. The downward Ad nAb trend that starts at 6 months continued until 24 months of age, but began to trend upwards at 36 months presumably in response to de novo infections. This indicates that the infant population would be considered naïve and receptive to Ad vectored vaccines using any of the 7 adenoviruses. 
A.
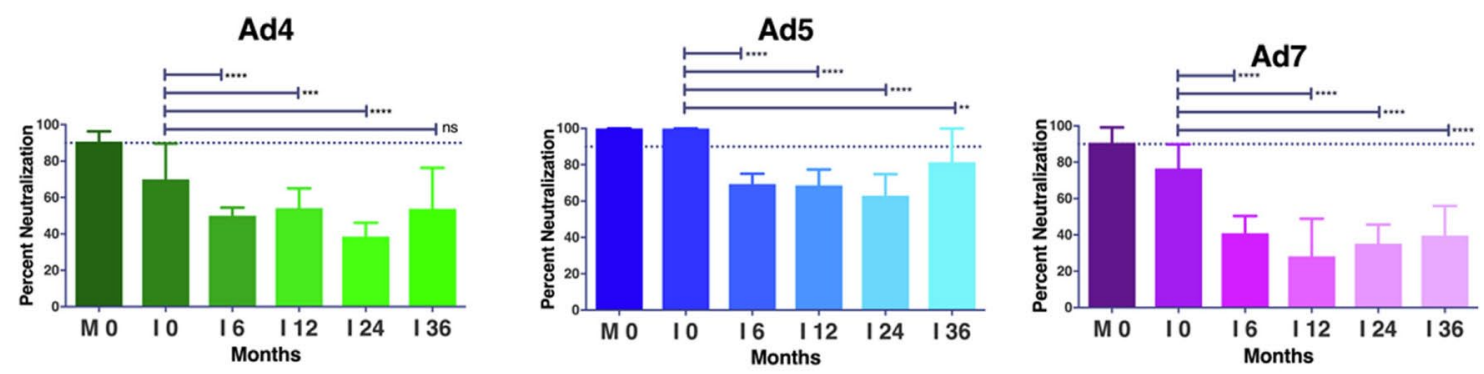

\section{Pre-ART}
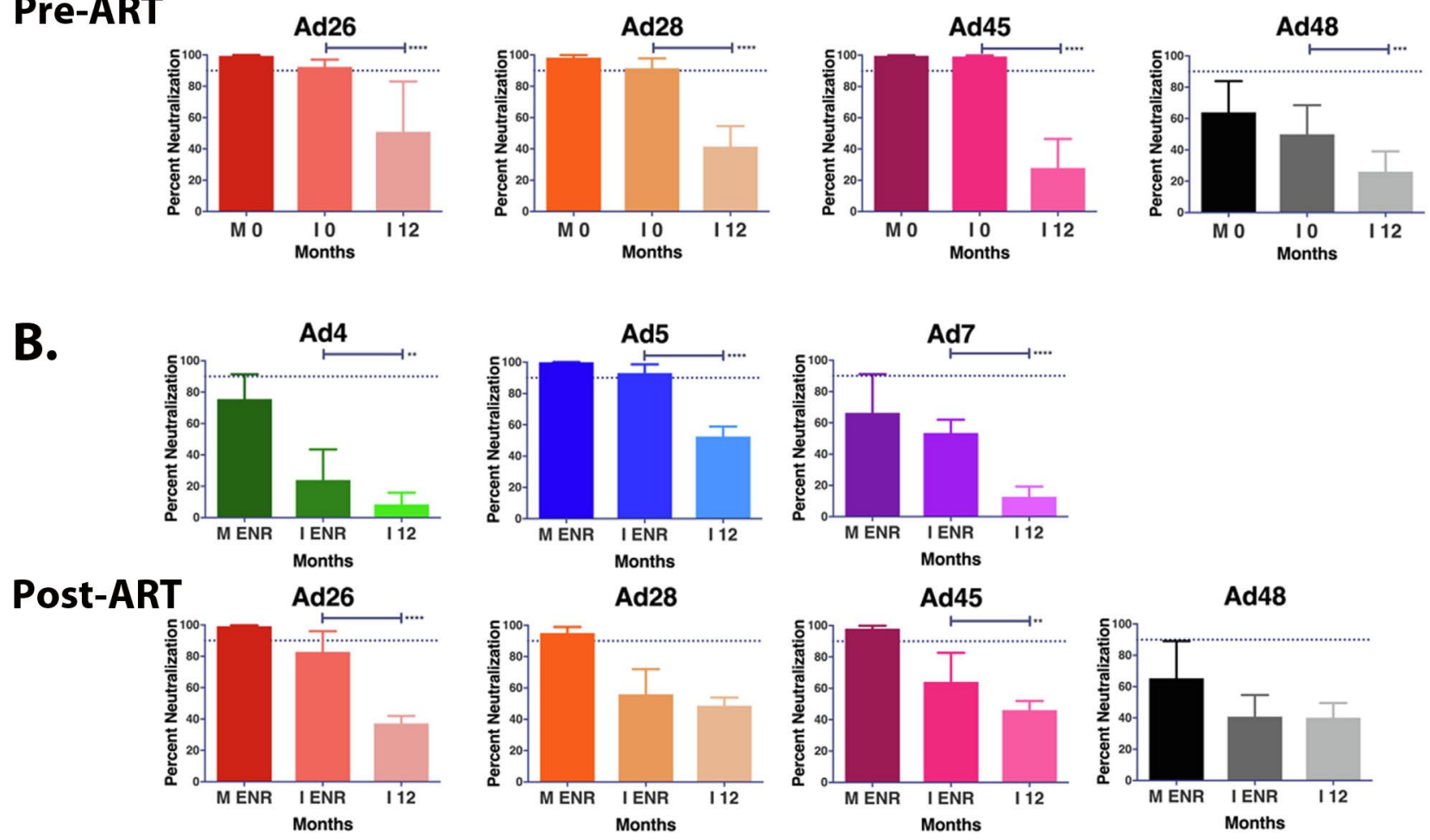

Fig. 5. Neutralizing antibody decay over time in Zambian infants. Decay in neutralizing antibodies was evaluated at o, 6, 12, 24 and 36 months for Ad4, Ad5, and Ad7 in the pre-ART cohort (A). Only the 12 month time point was analyzed for the preART (A) for Ad26, Ad28, Ad45 and Ad48 and all Ads for the post-ART cohort (B) due to sampling limitations. Significant change in average neutralization was evaluated as a comparison to o months or enrollment (ENR) by Mann-Whitney analysis $\left({ }^{*} \mathrm{P} \leq 0.05,{ }^{* *} \mathrm{P} \leq 0.001,{ }^{* * *} \mathrm{P} \leq 0.0001\right)$. 


\section{Discussion}

The WHO reports nearly 90\% of the world's malaria infections, nearly $25 \%$ of TB cases, and 70\% of HIV cases are located in Africa [2-4]. Many of the diseases that are prominent in the region either have no vaccine available or are of very low efficacy, high-cost or with cold-chain dependent options that need improvement. Children in SSA, and elsewhere, are often exposed to infectious diseases at a young age, which makes infant vaccination crucial for effective disease control and child well-being and development. Indeed, our lab and others have demonstrated that seroconversion to Kaposi's Sarcoma Herpesvirus (KSHV) occurs in sub-Saharan infants at levels near the adult prevalence by 4 years of age $[37,38]$. Using adenoviruses as a vaccine vector has been shown to elicit strong $\mathrm{T}$ and $\mathrm{B}$ cell responses to inserted transgenes and could also confer immunity to the Ad used as the backbone, thereby decreasing the burden of adenovirus associated diseases in young children. Adenoviruses are relatively easy to manipulate and can be designed to be replication deficient where only the inserted transgene will be expressed in infected cells. Unlike many vaccine platforms, Ad based vaccines would not require cold storage. In combination with the fact that they can be grown to high titers in vitro at relatively low cost, this makes Adenovirus an ideal vector for vaccines that could realistically be implemented on a large scale in Africa $[12,25]$.

While Adenoviruses have been studied for over 20 years for their use as a vaccine and gene therapy, it has been suggested that pre-exposure can lead to decreased efficacy of the vector $[14,19,20,39]$. For this reason, we investigated the seroreactivity profile of Ad within Zambia to establish a targeted approach for future vaccine strategies in this region of SSA. Other groups previously investigated neutralization against several of these Ads in sub-Saharan adults, but the results have been variable, and were not conducted longitudinally in children, the most likely recipients of vaccinations. In 2007, Abbink et al. reported seroreactivity to Ad5, Ad26, and Ad48 in SSA at 100\%, $21 \%$ and $3 \%$, respectively [25]. However, in 2011, Barouch et al. reported the South African adult Ad5, Ad26, and Ad48 seroprevalence to be $87.9-89.5 \%, 43.1-53.2 \%$, and $13.3-24.6 \%$, respectively [33]. Our results are in agreement with these reports for some Ad subtypes 
whereas for others, the responses in our cohorts are distinct from than those cited above. For example, while the median nAb responses to Ad 48 were also the lowest in both our cohorts, the magnitude of the response was 3-20 fold higher than the studies cited above, at $64 \%$ and $65.3 \%$ neutralization, respectively. In addition, low level neutralization was readily detected in infants born to these mothers, whereas previous studies suggested that little Ad nAb was detected in young children [33]. Nevertheless, the discrepant results could be a function of changes or differences in circulating Ad genotypes/serotypes in the region over time, or differences in experimental methods used for quantifying neutralization. For example, we see a decrease in maternal $\mathrm{nAb}$ responses against $\mathrm{Ad} 4$ and $\mathrm{Ad} 7$ between the pre- and postART cohorts which appears to correlate to a decrease in nAb transfer. This, however, does not explain the significant decrease in nAb transfer in the post-ART cohort against Ad5, Ad26, Ad28 or Ad45. The basis of methodological differences is also unclear since other studies quantified neutralizing responses by serial dilution but were unable to detect $\mathrm{nAb}$ at a 1:2 dilution, whereas we readily detected $\geq 90 \%$ neutralization at a 1:30 dilution of plasma.

Two other studies have investigated $\operatorname{Ad} 5$ seroprevalence in both HIV-1 positive and negative adult populations. Both reported no significant difference in Ad nAb between infected and uninfected populations $[40,41]$. These data suggest two possibilities. The first, and less likely, is that development of $\mathrm{Ad} \mathrm{nAb}$ responses are not impacted by alterations to the $\mathrm{CD} 4 \mathrm{~T}$ cell compartment since $\mathrm{T}$ cell depletion induced by HIV-1 infection did not diminish the neutralizing response. Alternatively, and perhaps more likely, the timing of infant de novo anti-adenoviral responses in our study suggests environmental exposure to adenoviral infection early in life, long before initiation of sexual activity. Thus, nAb responses had time to develop and mature immunological memory that likely is independent of $\mathrm{CD} 4 \mathrm{~T}$ cell help in anamnestic responses. This second interpretation is supported by the lack of any statistically significant differential in Ad $\mathrm{nAb}$ responses in the mothers in our cohort as a result of ART treatment (i.e. CD4 T cell reconstitution).

Our study is the first to longitudinally quantify the prevalence of $\mathrm{nAb}$ against a subset of Ads in infants starting at date-of-birth (DOB). This is a timepoint where nAbs are most likely derived prenatal 
transfer of maternal nAb. In the pre-ART cohort infants, we found significantly decreased nAb against Ad4, Ad7, Ad26, and Ad28, in comparison to the cognate mothers suggesting inefficient transfer or perhaps insufficient titer in the mothers despite high \% neutralization. In contrast, the infant DOB nAb levels to Ad5 and Ad45 approached adult levels, implying efficient transplacental transfer, whereas Ad48 $\mathrm{nAb}$ in infants was below 90\%. In the post-ART cohort, transfer of $\mathrm{nAb}$ against the investigated Ads was decreased. Of note, the median DOB percent neutralization of Ad5 was still high at 93\%, in contradiction to reports suggesting that infants lacked nAb against Ad5 [33].

A unique aspect of our study was the three-year follow-up of infants that allowed us to quantify the decay of maternally transferred $\mathrm{nAb}$ and to identify the timing of potential de novo responses to Ad infection. In both cohorts, median neutralization levels against all seven Ads was significantly lower by 12 months of age than at o months, and well below both the 90\% and 70\% threshold. Adenoviral infection and the resultant de novo humoral responses would explain the increases in infant $\mathrm{nAb}$ at 36 months seen against $\mathrm{Ad} 4$ and $\mathrm{Ad} 5$, this is consistent with post-weaning exposure to fecal-oral pathogens through solid food and increased social interactions. This study is the first to address the kinetics of nAb decay in infants where we potentially also identify exposure to the various Ads. Our findings implicate the existence of a window, between 12 and 24 months of age, that would support vaccination against regionally important pathogens without concern for preexisting immunity.

In this study, the changes in adult seroreactivity to seven different Adenoviruses as well as the decay of $\mathrm{nAb}$ in children were examined in order to establish a timeline for vaccination in Zambia. We found that none of the seven Ads should be used in adults without pre-existing immunity being a major consideration as most adults neutralized the virus. In contrast, children have a window for vaccination between 12 and 24 months of age where maternally transferred antibodies decrease, and de novo infection has not yet occurred. All seven of the investigated Ads would work for this purpose since all showed low seroreactivity; but Ad4 and Ad7 are the most promising candidates as they had the lowest seroprevalence in the post-ART infants, and have already been tested for efficacy against adult Ad4 and Ad7 diseases in the United States military. 
Acknowledgments - The authors would like to graciously thank the Zambian mothers and infants who were participants in this study. We also thank Ms. Brigette Corder for assistance in preparation of the final manuscript. This study was supported in part of PHS grants from the National Institute of Health; National Cancer Institute grants (Ro1 CA75903), National Cancer Institute/Fogarty International Program grant U54 CA221204-01, and National Institute of Allergy and Infectious Diseases Ruth L. Kirschstein National Research Service Award (T32 AI125207) to CW. SP is a Ruth L. Kirschstein fellow.

Declaration of Competing Interest - The authors declare that they have no known competing financial interests or personal relationships that could have appeared to influence the work reported in this paper.

\section{Appendix A. Supplementary material}
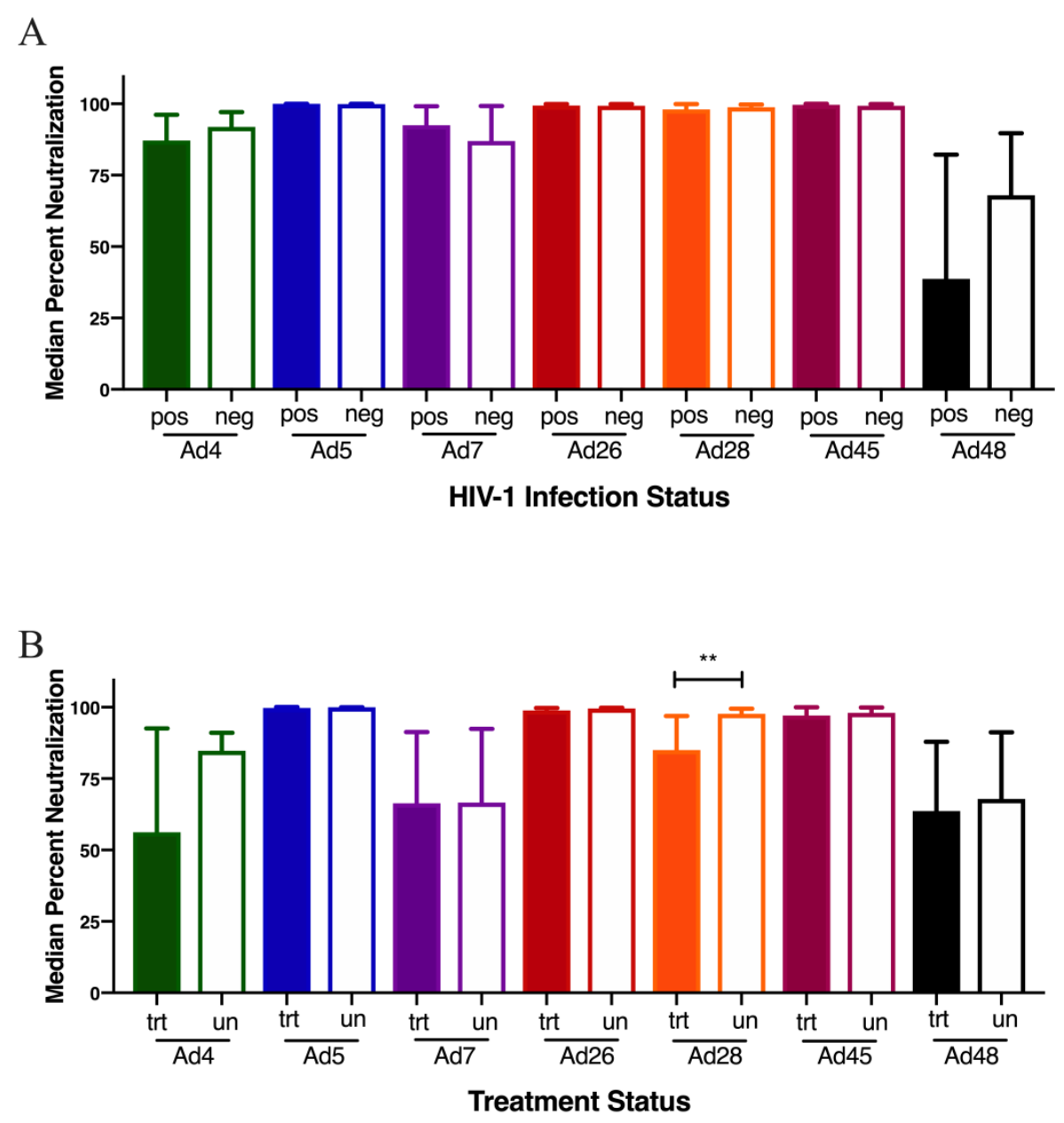

Supplemental Figure 1. Median percentage of neutralization in the mothers against all seven Ads was analyzed for significance based on HIV-1 infection status (A)or ART treatment status (B). ( $\left.{ }^{* *} \mathrm{P} \leq 0.01\right)$ 


\section{References}

[1] Anonymous. HIV/AIDS; 2018. https://www.who.int/news-room/fact-sheets/ detail/hiv-aids.

[2] Anonymous. Tuberculosis (TB); 2019. On World Health Organization. https:// afro.who.int/health-topics/tuberculosis-tb.

[3] Anonymous. WHO|10 facts on malaria; 2016. On World Health Organization. https://www.who.int/features/factfiles/malaria/en/.

[4] Anonymous. WHO|Data and statistics; 2018. On World Health Organization. https://www.who.int/hiv/data/en/.

[5] Chen RF, Lee CY. Adenoviruses types, cell receptors and local innate cytokines in adenovirus infection. Int Rev Immunol 2014;33:45-53.

[6] Alexander J, Ward S, Mendy J, Manayani DJ, Farness P, Avanzini JB, et al. Preclinical evaluation of a replication-competent recombinant adenovirus serotype 4 vaccine expressing influenza $\mathrm{H}_{5}$ hemagglutinin. PLoS ONE 2012;7:e31177.

[7] Stephenson KE, Keefer MC, Bunce CA, Frances D, Abbink P, Maxfield LF, Neubauer GH, Nkolola J, Peter L, Lane C, Park H, Verlinde C, Lombardo A, Yallop C, Havenga M, Fast P, Treanor J, Barouch DH. First-in-human randomized controlled trial of an oral, replicating adenovirus 26 vector vaccine for HIV-1. PLoS One 2018;13.

[8] Barry M. Single-cycle adenovirus vectors in the current vaccine landscape. Exp Rev Vaccin 2018;17:163-73.

[9] Weaver EA, Barry MA. Low seroprevalent species D adenovirus vectors as influenza vaccines. PLOS ONE 2013;8:e73313.

[10] Gach JS, Gorlani A, Dotsey EY, Becerra JC, Anderson CT, Berzins B, et al. HIV-1- Specific antibody response and function after DNA prime and recombinant adenovirus 5 Boost HIV vaccine in HIV-Infected subjects. PLoS ONE 2016;11: e0160341.

[11] Baden LR, Liu J, Li H, Johnson JA, Walsh SR, Kleinjan JA, et al. Induction of HIV-1-specific mucosal immune responses following intramuscular recombinant adenovirus serotype $26 \mathrm{HIV}-1$ vaccination of humans. J Infect Dis 2015;211:518-28.

[12] Bullard BL, Corder BN, Gorman MJ, Diamond MS, Weaver EA. Efficacy of a T cell-biased adenovirus vector as a Zika virus vaccine. Sci Rep 2018;8:18017.

[13] Geisbert TW, Bailey M, Hensley L, Asiedu C, Geisbert J, Stanley D, et al. Recombinant adenovirus serotype 26 (Ad26) and Ad35 vaccine vectors bypass immunity to $\mathrm{Ad}_{5}$ and protect nonhuman primates against ebolavirus challenge. J Virol 2011;85:4222-33.

[14] Dicks MD, Spencer AJ, Edwards NJ, Wadell G, Bojang K, Gilbert SC, et al. A novel chimpanzee adenovirus vector with low human seroprevalence: improved systems for vector derivation and comparative immunogenicity. PLOS ONE 2012;7:e40385.

[15] Zhu FC, Wurie AH, Hou LH, Liang Q, Li YH, Russell JB, et al. Safety and immunogenicity of a recombinant adenovirus type- 5 vector-based Ebola vaccine in healthy adults in Sierra Leone: a single-centre, randomised, doubleblind, placebo-controlled, phase 2 trial. Lancet 2017;389:621-8. 
[16] Choudhry A, Mathena J, Albano JD, Yacovone M, Collins L. Safety evaluation of adenovirus type 4 and type 7 vaccine live, oral in military recruits.

Vaccine 2016;34:4558-64.

[17] Fausther-Bovendo H, Kobinger GP. Pre-existing immunity against Ad vectors: humoral, cellular, and innate response, what's important? Hum Vaccin Immunother 2014;10:2875-84.

[18] Nwanegbo E, Vardas E, Gao W, Whittle H, Sun H, Rowe D, et al. Prevalence of neutralizing antibodies to adenoviral serotypes 5 and 35 in the adult populations of the Gambia, South Africa, and the United States. Clin Diagn Lab Immunol 2004;11:351-7.

[19] Alonso-Padilla J, Papp T, Kajan GL, Benko M, Havenga M, Lemckert A, et al. Development of novel adenoviral vectors to overcome challenges observed with HAdV-5-based constructs. Mol Ther 2016;24:6-16.

[20] Buchbinder SP, Mehrotra DV, Duerr A, Fitzgerald DW, Mogg R, Li D, Gilbert PB, Lama JR, Marmor M, Del Rio C, McElrath MJ, Casimiro DR, Gottesdiener KM, Chodakewitz JA, Corey L, Robertson MN. Step Study Protocol TEfficacy assessment of a cell-mediated immunity HIV-1 vaccine (the Step Study): a double-blind, randomised, placebo-controlled, test-of-concept trial. Lancet 2008;372:1881-93.

[21] Flasche S, Jit M, Rodriguez-Barraquer I, Coudeville L, Recker M, Koelle K, et al. The long-term safety, public health impact, and cost-effectiveness of routine vaccination with a recombinant, live-attenuated dengue vaccine (DENGVAXIA): a model comparison study. PLoS Med 2016;13:e1002181.

[22] Halstead SB. Dengvaxia sensitizes seronegatives to vaccine enhanced disease regardless of age. Vaccine 2017;35:6355-8.

[23] Pauly M, Hoppe E, Mugisha L, Petrzelkova K, Akoua-Koffi C, Couacy-Hymann E, et al. High prevalence and diversity of species D adenoviruses (HAdV-D) in human populations of four Sub-Saharan countries. Virol J 2014;11:25.

[24] de Andrade Pereira B, Maduro Bouillet LE, Dorigo NA, Fraefel C, BrunaRomero O. Adenovirus specific pre-immunity induced by natural route of infection does not impair transduction by adenoviral vaccine vectors in mice. PLOS ONE 2015;10:e014526o.

[25] Abbink P, Lemckert AA, Ewald BA, Lynch DM, Denholtz M, Smits S, et al. Comparative seroprevalence and immunogenicity of six rare serotype recombinant adenovirus vaccine vectors from subgroups B and D. J Virol 2007;81:4654-63.

[26] Duffy MR, Alonso-Padilla J, John L, Chandra N, Khan S, Ballmann MZ, et al. Generation and characterization of a novel candidate gene therapy and vaccination vector based on human species D adenovirus type 56. J Gen Virol 2018;99:135-47.

[27] Farrow AL, Peng BJ, Gu L, Krendelchtchikov A, Matthews QL. A novel vaccine approach for Chagas disease using rare adenovirus serotype 48 vectors. Viruses 2016;8:78.

[28] Lynch 3rd JP, Kajon AE. Adenovirus: epidemiology, global spread of novel serotypes, and advances in treatment and prevention. Semin Respir Crit Care Med 2016;37:586-602. 
[29] Scott MK, Chommanard C, Lu X, Appelgate D, Grenz L, Schneider E, et al. Human adenovirus associated with severe respiratory infection, Oregon, USA, 2013-2014. Emerg Infect Dis 2016;22:1044-51.

[30] Mayindou G, Ngokana B, Sidibe A, Moundele V, Koukouikila-Koussounda F, Christevy Vouvoungui J, et al. Molecular epidemiology and surveillance of circulating rotavirus and adenovirus in Congolese children with gastroenteritis. J Med Virol 2016;88:596-605.

[31] Weaver EA. Vaccines within vaccines: the use of adenovirus types 4 and 7 as influenza vaccine vectors. Hum Vaccin Immunother 2014;10:544-56.

[32] Abbink P, Maxfield LF, Ng'ang'a D, Borducchi EN, Iampietro MJ, Bricault $\mathrm{CA}$, et al. Construction and evaluation of novel rhesus monkey adenovirus vaccine vectors. J Virol 2015;89:1512-22.

[33] Barouch DH, Kik SV, Weverling GJ, Dilan R, King SL, Maxfield LF, et al. International seroepidemiology of adenovirus serotypes 5, 26, 35, and 48 in pediatric and adult populations. Vaccine 2011;29:5203-9.

[34] Yang WX, Zou XH, Jiang SY, Lu NN, Han M, Zhao JH, et al. Prevalence of serum neutralizing antibodies to adenovirus type 5 (Ad5) and 41 (Ad41) in children is associated with age and sanitary conditions. Vaccine 2016;34:5579-86.

[35] Zhang X, Wang J, Lu J, Li R, Zhao S. Correction to: Immunogenicity of adenovirus-vector vaccine targeting hepatitis $B$ virus: non-clinical safety assessment in non-human primates. Virol J 2018;15:137.

[36] Lion T. Adenovirus infections in immunocompetent and immunocompromised patients. Clin Microbiol Rev 2014;27:441-62.

[37] Nalwoga A, Miley W, Labo N, Elliott A, Cose S, Whitby D, et al. Age of infection with Kaposi sarcoma-associated Herpesvirus and subsequent antibody values among children in Uganda. Pediatr Infect Dis J 2018;37:e225-8.

[38] Olp LN, Minhas V, Gondwe C, Poppe LK, Rogers AM, Kankasa C, et al. Longitudinal analysis of the humoral response to Kaposi's sarcomaassociated herpesvirus after primary infection in children. J Med Virol 2016;88:1973-81.

[39] Saxena M, Van TT, Baird FJ, Coloe PJ, Smooker PM. Pre-existing immunity against vaccine vectors-friend or foe? Microbiology 2013;159:1-11.

[40] Kostense S, Koudstaal W, Sprangers M, Weverling GJ, Penders G, Helmus N, et al. Adenovirus types 5 and 35 seroprevalence in AIDS risk groups supports type 35 as a vaccine vector. Aids 2004;18:1213-6.

[41] Sun C, Zhang Y, Feng L, Pan W, Zhang M, Hong Z, et al. Epidemiology of adenovirus type 5 neutralizing antibodies in healthy people and AIDS patients in Guangzhou, southern China. Vaccine 2011;29:3837-41. 